\title{
Analysis of Clinical Presentation And Management of Diabetic Foot Ulcer Cases with Preventive Measures in A Tertiary Care Hospital. Government Medical College And Esi Hospital. Coimbatore
}

\author{
${ }^{* 1}$ Dr. V. Lekshmi Narayani MS (GS), ${ }^{2}$ DGO Dr. Geetha MD \\ Associate Prof. Of Surgery GMC \& ESI Hospital, Coimbatore, Tamilnadu, India. \\ Associate Prof. Of Medicine GMC \& ESI Hospital, Coimbatore, Tamilnadu, India \\ *Corresponding author: Dr. V. Lekshmi Narayani *
}

\begin{abstract}
:
Aim: To study the age, sex, clinical presentation and management of diabetic foot ulcer cases attending referral hospital-GMC and ESI Hospital. Coimbatore. Analysing whether regular treatment and awareness of diabetic mellitus help in preventing complications.

Methods: A prospective study during a period of 6 months from NOVEMBER 2016-APRIL 2017 done in patients attending surgical department of GOVERNMENT MEDICAL COLLEGE AND ESI HOSPITAL, COIMBATORE.

Results: On evaluating the diabetic foot ulcer patients even with regular treatment there is no change in occurance of foot ulcer as the cause is mainly hyperglycemia along with infection in the normal as well as atheromatous changes in the vascular system.

Conclusion: Diabetic foot ulcer may be prevented by educating the patient on foot care as well asgoodglycemic control.

Keywords: Diabetes, debridement, foot ulcer, glycemic control, sensitive antibiotics.

\section{Introduction}

Diabetic foot ulcers are a complication of diabetes and approximately 5\% of sufferers require major amputation. Diabetes is the most common disease process associated with lower limb amputation accounting for approximately half of non traumatic amputations.(1)Diabetic foot ulcers result from neuropathy, vasculopathy, and immunopathy.(2) More than $60 \%$ of diabetic foot ulcers are primarily due to an underlying neuropathy.(2)Loss of nerve function is associated with hyperglycemia as reflected in the mean level of glycosylated Hb.overtime.Peripheral neuropathy affects all components of the peripheral nervous system, sensory, motor, and autonomic each of which contribute to foot ulcer development.(2,3) Self care is fundamental in diabetes management and prevention. Existing guideline state the need for patient education as a prerequisite to prevent ulceration.(4) Approximately $15 \%$ of people with diabetes will be affected by a foot ulcer during their lifetime.(5) Five year recurrence rates of foot ulcers are 70\%(6).Upto $85 \%$ of all amputations in relation to people with diabetes are preceeded by a foot ulcer.(5,6)People with diabetes with one lowerlimb amputation have a 50\% risk of developing a serious lesion in the second limb within 2 years.(7)People with diabetes have a 50\%mortality rate in the 5 years following the initial amputation. Conceptual frame work showing possible predictors of diabetic foot ulcer.
\end{abstract}




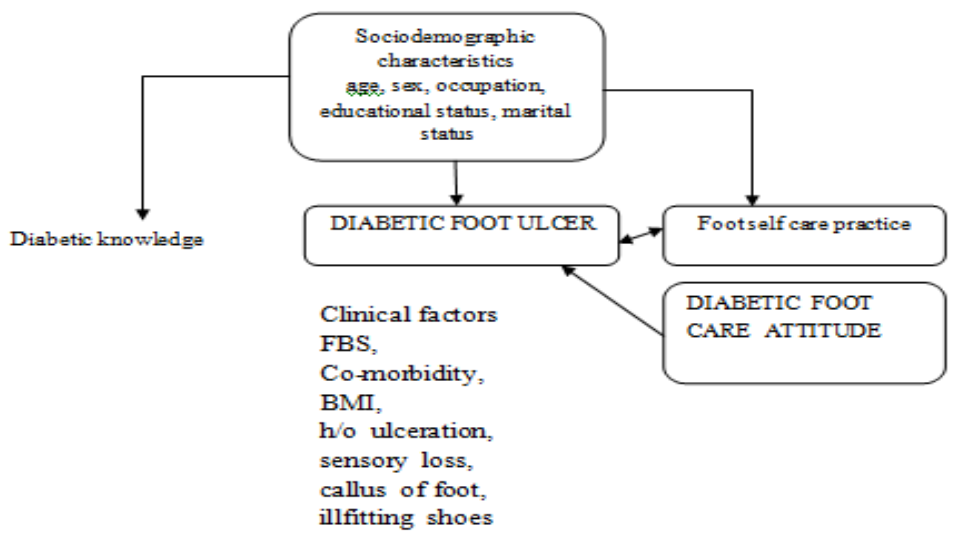

Aetiology And Classification Of Diabetic Foot Ulcers

The incidence of neuroischaemic ulcers is $52.3 \%$, neuropathic ulcers $36 \%$ and ischemic ulcers $11.7 \%$.Neuropathy includes sensory, motor, and autonomic components.

Deficient protective sensation leads to ulceration on high pressure areas, motor neuropathy results in biomechanical abnormalities an autonomic neuropathy causes decreased sweating and dry skin which is more prone to hyperkeratosis an ulceration. Ischeamia is caused by peripheral arterial disease resulting in decreased blood supply and tissue perfusion which significantly compromise ulcer healing. Peripheral arterial disease in patients with Diabetes tends to be multifocal and commonly affects the infrapoplitealvessels.Furthermore disease progression is usually accelerated(8) and together with microvascular disease results in poor outcomes.Endothelial dysfunction appears to be the main cause of both the macrovascular and microvascular disease and the mechanisms which contributes to this include persistanthyperglycemia, increased advanced glycation end products, oxidative stress, endothelial imflammation and reduced nitric oxide activity.

\section{WAGNER CLASSIFICATION FOR DIABETIC FOOT ULCER}

GRADE DISCRIPTION OF ULCERS

0 Intact skin in patients who are at risk

1 superficial ulcers with exposed subcutaneous tissue

2 Exposed tendon and deep structure

3 Ulcers extend to the deep tissue and have either associated soft tissue abscess or osteomylities

4 Ulcers include feet with partial gangrene.

5 Feet ulcers with more extensive gangrenous tissue.

Methodology-men and materials:

Allthe patients who attended surgery department of GMC AND ESI HOSPITAL, refered from ESIdispensary for diabetic foot ulcer were examined.Total no of patients 80 for a period of 6 months from NOVEMBER 2016-APRIL 2017.

Type Of Study.-Prospective study.

Inclusion criteria: patients of more than 18 years of age with diabetes having foot ulcer.

Patient recently diagnosed as DM with foot ulcer. Exclusion Criteria; Patients less than 18 years with foot ulcer, pregnant women, patient with septecimia.

Type Of Study: Prospective Study Observation \& Result :1 Sex Total no of patients was 80 .out of which Male 42(52\%) and Female 38.(48\%)

\section{Age of patient}

Among Male patients 18 were in the age group of 51-to 60, 12 were $61-70 \mathrm{yrs}, 8 \mathrm{pts}$ were $41-50 \mathrm{yr}$ age, more than 70 years 2 patients and 2 pts were below 40 yrs. Among female pts 14 were in the age group of 51-60 yrs, 8 were 71-80,6pts were in the age group of 61-706 pts were in the age group of 41-50 yrs and 4 pts were below 40 yrs.

\begin{tabular}{|l|l|l|}
\hline AGE & MALE & FEMALE \\
\hline $31-40$ & 2 & 4 \\
\hline $41-50$ & 8 & 6 \\
\hline $51-60$ & 18 & 14 \\
\hline $61-70$ & 12 & 6 \\
\hline $71-80$ & 2 & 8 \\
\hline
\end{tabular}




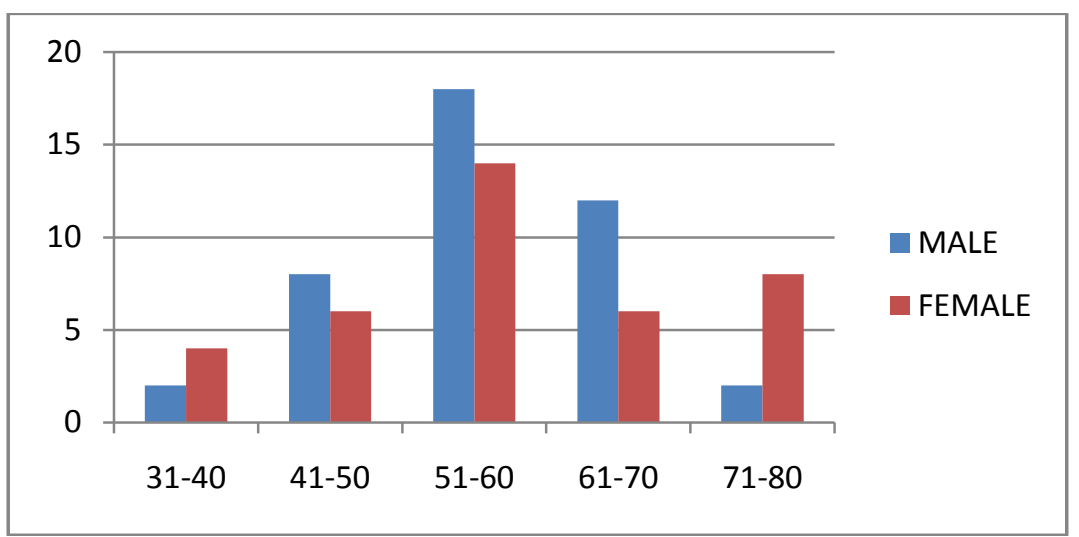

Whether they are ip or nip: total no of insured patients (ip)16 and non insured person were (nip) 64 .

Among ip, male were 12 and female 4.

Among the nip male pts 30 and 10 were working in private concern

Education: male female

Illiterate $\quad 4 \quad 4$

School $\quad 36 \quad 32$

College $\quad 2 \quad 2$

Total $\quad 42 \quad 38$

Residence

From Coimbatore district 54 patients and 26 patients came from nearby places like Thiruppur 8, Karur2, Palladam4, Pallakkad2, annur2, salam2, Madurai2, Pollachi2\& Dharmaburi2.

\section{Duration of illness:-}

Ranged from 3Days to 2Months. With Foot ulcer \& some have taken treatment either in ESI dispensaryor from CMCH or Pvt hospital.

Site of lesion:- The lower limb ulcer patients were taken for the study.
Lower Limb
RT
LT
24
BOTH LEGS
52
4

Type of lesion:-Patients were presented with superficial ulcer, deep ulcer, abscess, cellulitis with ulcer and pregangrenous or gangrenouschanges of the toes.

Ulcer

48

Gangrene

4

Cellulitis with ulser

28

\section{Investigations:-}

The patients were investigated with RBS, FBS, PPBS, BLOOD UREA, Serum creatinine HbA1C, X ray chest, $\mathrm{X}$ ray foot $\mathrm{AP}$ and lateral if needed, ECG.

HbA1c ranges from 7.4 to 9.8

RBS ranges from 100-200mgin 12 patients and 201-300 mgms\% in 68 patients.

\begin{tabular}{|l|l|l|}
\hline Fbs & Male & Female \\
\hline$<100$ & 6 & Nil \\
\hline $101-200$ & 25 & 23 \\
\hline $201-300$ & 10 & 11 \\
\hline $301-400$ & Nil & 4 \\
\hline$>400$ & 1 & Nil \\
\hline Total & 42 & 38 \\
\hline
\end{tabular}




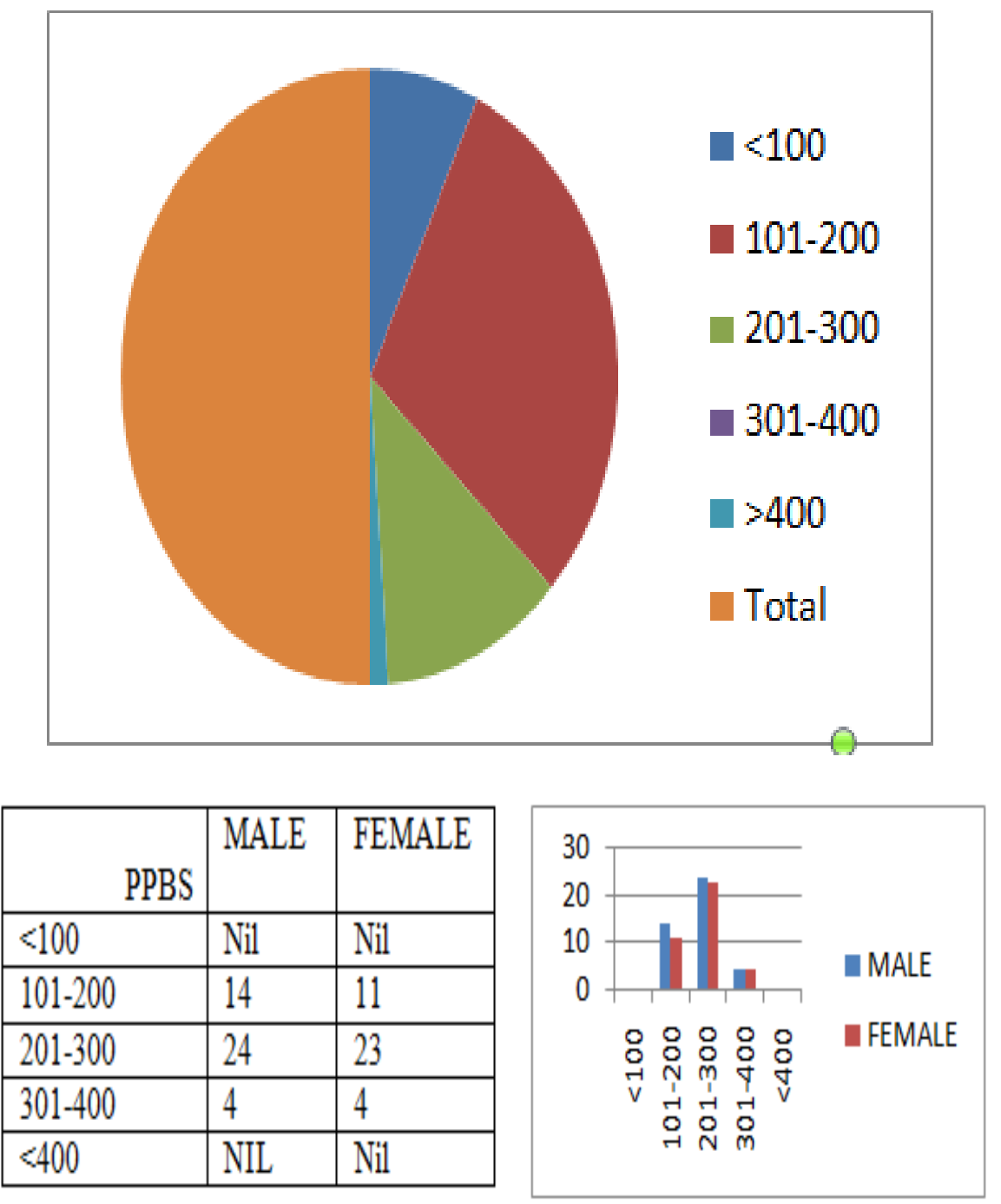

Blood sugar level of our patients was high showing that hyper glycemia is one of the most important factor for development of the ulcer.

Blood urea

Range

21-30mgms $\%$

$31-40$

41-50

$>50$
No.of patients

24

1

2

2 patients With blood urea $>50$ were treated and repeat blood urea came down to normal level after 5 days.

\section{Serum Creatinine}

$\begin{array}{ll}<1 \mathrm{Mgs} \% & 58 \\ 1.1 \mathrm{Mgs} \% \text { to } 2 \mathrm{Mg} & 20 \\ >2.1 \mathrm{Mgs} \text { to } 3 & 2\end{array}$

\section{Treatment}

After getting diabetalogist's opinion, the patients were treated with insulin \& oral hypoglycemic agents to bring the blood sugar under control. For the infection all pts were initially started on appropriate antibiotics includinganaerobic agents. Where the response nervosa was poor. Pus culture anaerobic agents sensitivity was done. According to fertility the antibiotics were given dimension of the antibiotics therapy ranged from 7days to 10 days. Most of the results showed that the micro organism was pseudomonas group. All the patients were asked the questions to know whether they were aware of complication of diabetes.

1. whether they are IP are NIP 
2. whether the foot ulcer was treated earlier either in ESI Dispensary or Private Concerns.

3. How long the pts having DM?

4. whether the pts adapt diet control

5. Are they do regular exercise?

6. Are they on regular treatment

\section{The Patients Are As Follows}

1. Among the total 80pts Insured Patients (IP) is $16(20 \%)$ and non Insured Patients (NIP) dependent on IP is 64. $(80 \%$

2. Awareness on dietary management in diabetics.

\begin{tabular}{|l|l|}
\hline Days & 08 \\
\hline$<11$ months & 04 \\
\hline $1-10$ years & 50 \\
\hline $11-20$ years & 10 \\
\hline $21-30$ years & 06 \\
\hline $31-40$ years & 02 \\
\hline Total & 80 \\
\hline
\end{tabular}

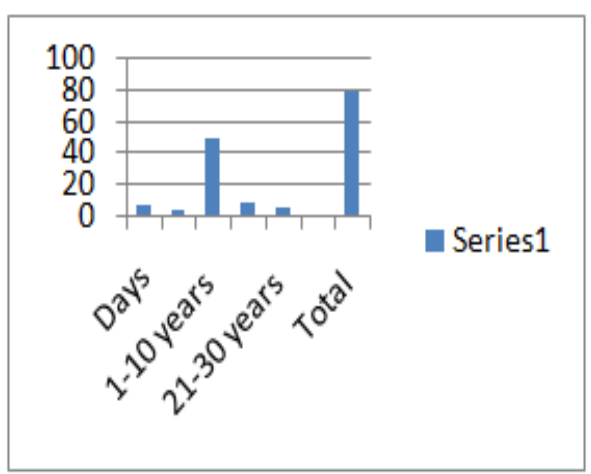

3. Whether treated for foot ulcer in Esi Dispensary or Private concern?

Most of the patients were treated and referred from Esi Dispensary. Two of the patients were treated in $\mathrm{CMCH}$ for one month, 4 patients got native treatment for foot ulcer wear which worsened the disease. 8 patients came to our hospital for the first consultation for foot ulcer.

4. How long DM known to the Patients?

5.

Newly diagnosed 42 male and 2 female

New diagnosed 04

1 month - 11 month 10

$1-5$ years 40

$6-10$ years 16

$11-15$ years $\quad 08$

$16-20$ years $\quad 02$

Total 80

6. Weather on diet control :-

50 pts are not on diet control.

30 pts are on diet control.

7. Weather do regular exercise 70 patients are not doing regular exercise.

10 patient only on regular exercise.

8. Weather patients on regular treatment?

50 pts are on regular drugs

26 pts are not on regular drugs

4 patients are newly deducted after coming to our hospital.

Weather already any surgical procedure like disarticulation or amputation done

Male $\quad 18$

Female $\quad 06$

24

Total 24 patients were already had some kind of surgical procedure for foot ulcer. Among male patients fore foot amputation done. 1year back

Left great toe amputation done. 2years back

Left Ak amputation done. 2years back

Left GT amputation done. 2years back

Rt. Great toe amputation 10 years back

Rt. little toe amputation 5 years back 
Rt. $4^{\text {th }}$ toe amputation 5 months back

Rt. $4^{\text {th }} \& 5^{\text {th }}$ toe amputation in ESIH.

Rt. Great toe amputation in August 2016

Rt. PT 3 disarticulation 1 year back.

Rt. BK amputation in ESIH.

\section{Among the female patient}

Great toe right amputation done in ESIH

Right great toe amputation done in ESIH

Left great toe amputation done in 2 years back

Diabetic Retinopathy was detected in 2 male patients who complaint of loss of vision.

Venous Doppler was done in 4 female patients having varicosity of vein with ulcer And found to be SFJ incompetent reflux. Varicosity in medial aspect of leg. Incompetent Perforator noted in medical aspect of leg, in below knee, lateral and middle level. No DVT. SPJ Normal.

Arterial Doppler done in patients with chronic ulcer and with gangrenous change. Arterial Doppler was found to be normal in 20 patients.

DPA Biphasic others normal in 5 patients. Diffuse Atheromatous wall thickening with proximal Vessels showing significant occlusion with distal vessels showing Biphasic flow in 2 patients.

Diffuse wall thickening noted in left LL arterial system in one patient.

\section{Discussion}

Total no. of patients with diabetic foot ulcer attended surgical op \& got admitted as in-patients in GMC \& ESIH was 80 during the period NOV 2016 -APRIL 2017 (6 months).

1. Among the 80 patients male was $42(52 \%)$ and female was $38(48 \%)$. There is not much sex

2. Variation regarding.

3. The most common age group in male with DM \& foot ulcer was $51-60 \mathrm{yrs}$ as $18(45 \%)$ patients

4. \& 61-70yrs as $12(28.57 \%)$ patients fall in this group. Whereas as in female the common age group was 5160yrs with $14(36.8 \%)$ patients and 71-80yrs with $8(21.05 \%)$ patients.

5. 3. Among 80 patients insured patients were $16(20 \%)$ and non insured patients (dependent patients ) were $64(80 \%)$ showing that there is ESI benefit for dependents.

6. 4. Educated male patients are 38, graduate 2 patients and illiterate 4 patients showing 36 have attended school and at least studied primary school. Among the female patient's 34 patients are educated 4 illiterate and 2 patients graduated.

7. 5. Among the 80 patients 54(67.5\%) patients were residing in Coimbatore district and referred from ESI Dispensary 26 patients were outside Coimbatore from nearby places. 26 patients are from Tirupur 8, Karur 4, Palladam 4 , Palakkad 2, Annur 2 , Selam 2, Madurai 2, Pollachi 2, and Dharmapuri 2.

8. Duration of illness ranges from 3 days to 2 months.

9. Site of lesion in the lower limb showed that right leg ulcer was 52 patients $(65 \%)$ left leg ulcer was in 24 patients $(30 \%)$ and both leg ulcers was in 2 patients $(5 \%)$.

10. Types of lesion showed that diabetic foot ulcer was present in 48 patients (60\%) gangrene in 4 patients $(5 \%)$ cellulitis with diabetic foot ulcer in 28 patients $(35 \%)$.

\section{Investigations}

Urine sugar albumin and deposit was done in all the patients. In 68 patients sugar was >++ and in 12 patients urine sugar was + . Urine albumin ranges from trace and nil as the patients with high blood urea serum creatinine showed albumin in the urine. The urine albumin +ve in 30 patients and in 50 patients there is no albumin in the urine. RBS ranges from $100 \mathrm{mgm}-200 \mathrm{mgm}$ in 12 patients and 201mgm-300mgm in 68 patients. Fasting \& blood sugar was done on the next day which showed that the patients are not under control of the blood sugar. 


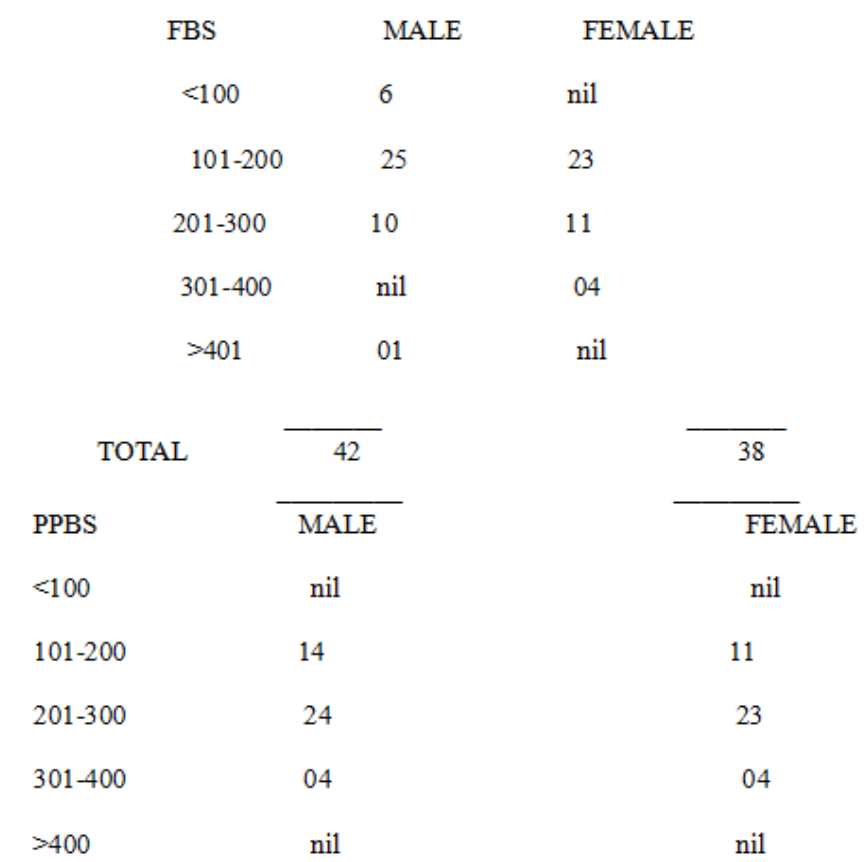

This showed that patients with blood sugar in the range of 201-300mgm are more prone to develop leg ulcers 24 patients male, 23 patients female showing 47 patients among 80 were in this range.

$\begin{array}{lcc}\text { Blood urea } & & \\ 21-30 \mathrm{mgm} \% & 50 & 62.50 \% \\ 31-40 \mathrm{mgm} \% & 24 & 30 \% \\ 41-50 \mathrm{mgm} \% & 04 & 5 \% \\ >50 & 2 & 2.5 \%\end{array}$

Serum creatinine

$\begin{array}{ccc}<1 \mathrm{mgm} \% & 58 \text { patients } & 72.5 \% \\ 1.1 \mathrm{mgm}-2 \mathrm{mgm} & 20 & 25.5 \% \\ 2.1 \mathrm{mgm}-3 \mathrm{mgm} & 2 & 2.5 \% \\ \text { HbAc ranges from } & 7.4 \text { to } 9.8 & \\ 6-7 & \text { NIL } & \\ 7.1-8 & 58 & 72.5 \% \\ 8.1-9 & 20 & 25.5 \% \\ 9.1-10 & 2 & 2.5 \%\end{array}$

Showed that 58 patients (72.5\%) with 7.1-8 ranges of HbA\& 20 patients (25.5\%) with 8.1-9, 2 patients with 9.1 -10. This shows that patients with poor long term control of blood sugar were more prone for DM ulcer.

The beneficiaries are dependants parents 64 patients $(80 \%)$ and 16 patients $20 \%$ are insured patients. Most of the patients were referred from ESI dispensary. In analysing the duration of DM 40 patients diagnosed $\leq 5$ years back, 16 patients $\leq 10 \mathrm{yrs}, 10$ patients diagnosed $\geq 11$ months \& 2 patients were $<20$ yrs . Newly diagnosed patients 4 ( 2 male \& female ) 50 patients were on strict diet control 30 patients were not on diet control. 7 patients were doing regular exercise and 10 patients were not doing regular exercise. 50 (62.5\%)patients were on regular drugs, 26 patients $(32.5 \%)$ were not on regular drugs, 4 patients $(5 \%)$ were newly diagnosed. This analysis showed that even with regular treatment the blood sugar was not under control and hyperglycemia was prone to develop food ulcers. In 24 patients (40\%) already some surgical procedure was done in the other limb or same limb. Diabetic Retinopathy was detected in 2 patients. Venous Doppler done showed that 4 patients (5\%) had varicosity of veins . Arterial Doppler done in gangrenous foot chronic ulcer patient showed normal flow in 20 patients. DPA biphasic others normal in 5 patients. Diffuse athermatous wall thickening with proximal vessels showing significant occlusion with distal vessels showing Biphasic flow in 2 patients diffuse wall thickening noted in left LL arterial system in 1 patient. 


\section{Conclusion}

DM in the disease affecting other organs with micro and macro angiopathy changes even with patient on regular diet control, exercise and treatment if the blood sugar level is not under control. The most common age group affected are $51-60$ yrs even in both sex. Right lower limb ulcer is more common. Diabetic ulcer foot is more common duration of diabetes mellitus is $1-5$ yrs.

Patients need to be educated on foot care. During every visit to the Diabetologist, physician, examination of the distal pulses, changes in skin colour over the feet, examination for neurological changes and presents of tropic ulcers is mandatory. This approach can definitely reduce the instances of diabetic foot ulcer and the probability of amputations.

\section{References}

[1]. Doulton A.J.M. Vikikyll,LRagnarson- Tennvall G and Apelgovist J(2005). The global burden Of diabetic foot disease Lamert,366,1719-1724 doi 10. 1016/s 0140 - 6736 (05) 67698-2

[2]. Bowering CK (2001) Diabetic foot ulcers patho physiology, assessment and therapy.Canadian family physician 47, $1007-1016$.

[3]. Clair D (2011) Diabetic foot ulcers / assessment for the long term living and education continuingcome professional, 60, 20-22.

[4]. International working group on the diabetic foot. International contractive on the diabetic foot. Contractive section of international diabetic federation 2007.

[5]. Bakker K Riley p(2005) the year of the diabetic foot. Diabetic voice 50:11-14

[6]. International making group on the diabetic foot. International consensus on the diabetic foot 1993, 2003.

[7]. Jude (2001) assessment of diabetic foot. Chronic mound case. A clinical source look far health care professionals ( $3^{\text {rd }}$ edtn) HMP communication inc589-597.

[8]. Dormandy J, Heack I, VigS.Prdicting which patients nil develop chronic critical by ischemia semin vase surg 1999; 12:138-41 